TECHNICAL NOTE

\author{
J.B. White \\ K.F. Layton \\ A.J. Evans \\ F.C. Tong \\ M.E. Jensen \\ D.F. Kallmes \\ J.E. Dion \\ H.J. Cloft
}

\section{Transorbital Puncture for the Treatment of Cavernous Sinus Dural Arteriovenous Fistulas}

SUMMARY: This report describes a series of patients for whom dural arteriovenous fistulae (DAVFs) of the cavernous sinus were successfully embolized using a percutaneous, transorbital technique to directly cannulate the cavernous sinus. A vascular access needle and catheter are percutaneously advanced along the inferolateral aspect of the orbit to access the cavernous sinus via the superior orbital fissure. Safe and effective embolization is achieved without the need for a surgical cut-down.
C arotid cavernous fistulas (CCFs) are arteriovenous shunts that are fed by the external carotid artery, the internal carotid artery (ICA), or both and drain by way of the cavernous sinus and its tributaries. The surgical treatment of cavernous sinus dural arteriovenous fistulas (DAVFs) is technically difficult and associated with significant morbidity. ${ }^{1,2}$ As such, endovascular embolization via a transvenous approach has become a mainstay of treatment. ${ }^{3}$ Numerous transvenous approaches have been described, with most practitioners preferring a transfemoral access. ${ }^{4}$ However, when the veins are not traversable from a transfemoral route, many have advocated a surgical exposure of the superior ophthalmic vein followed by direct catheterization of the exposed vein. ${ }^{4}$

An alternative approach for endovascular treatment is through a direct transorbital puncture of the cavernous sinus or indirectly via the inferior ophthalmic vein (IOV). We are aware of only 1 other account in the literature that describes an IOV approach. ${ }^{5}$ The purpose of this report is to describe the successful embolization of a series of patients with CCFs by using a direct transorbital cavernous sinus or IOV puncture to treat fistulas. We have found that that this approach is safe and technically straightforward while providing a useful alternative to other transvenous routes.

\section{Patients and Techniques}

This study was approved by the Mayo Foundation Institutional Review Board. Eight patients presenting between 2002 and 2006 were diagnosed with CCFs requiring endovascular embolization. In each case, the patient consented to an angiogram with the intent to treat the CCF. Using a sterile technique, we accessed the common femoral artery, and a $5 \mathrm{~F}$ sheath was placed. A $5 \mathrm{~F}$ vertebral angiography catheter was then introduced into the appropriate carotid artery for baseline arterial digital subtraction angiography (DSA). Once the arterial and venous anatomy of the CCF was visualized, transfemoral venous access was then obtained with the intent of treating via the inferior petrosal sinus route. In each patient, the transfemoral venous ap-

Received February 28, 2007; accepted after revision April 2

From the Departments of Neurologic Surgery (J.B.W.) and Radiology (K.F.L., D.F.K., H.J.C.), Mayo Clinic, Rochester, Minn; the Department of Radiology (F.C.T., J.E.D.), Emory University, Atlanta, Ga; and the Department of Interventional Neuroradiology (A.J.E., M.E.J.), University of Virginia Health System, Charlottesville, Va.

Please address correspondence to Harry J Cloft, MD, Department of Radiology, Mayo Clinic, 200 First St SW, Rochester, MN 55905; e-mail: cloft.harry@mayo.edu

DOI 10.3174/ajnr.A0663 proach was not possible, and access by way of direct transorbital puncture was pursued.

With the patient under general anesthesia, the eye region was prepped in a sterile fashion. Using continuous road-mapping and fluoroscopic guidance after injection of the carotid artery catheter, we placed a 6-inch 18-gauge radial arterial-line catheter into the orbit, inferolateral to the globe. Access was obtained by direct needle puncture with gradual advancement along the inferior orbital rim (Fig 1). With an oblique projection for the anteroposterior fluoroscopy tube, the superior orbital fissure (SOF) was easily separated from the optic canal. During advancement of the needle along the orbital floor, it is important to avoid the optic canal as the SOF is entered to prevent injury to the optic nerve. As the needle approaches the SOF under biplane fluoroscopy, frequent removal of the inner stylet was performed after the needle was advanced in 1-mm increments. Once arterialized flow was observed, venography was then performed.

Using a coaxial technique, we advanced a microcatheter over a guidewire into the cavernous sinus. Coil embolization was subsequently performed. The fistula was densely packed until cessation of fistula flow was noted, and the status of fistula flow was easily monitored with the arterial catheter that had already been placed. The transorbital catheter and the transfemoral catheter were then removed.

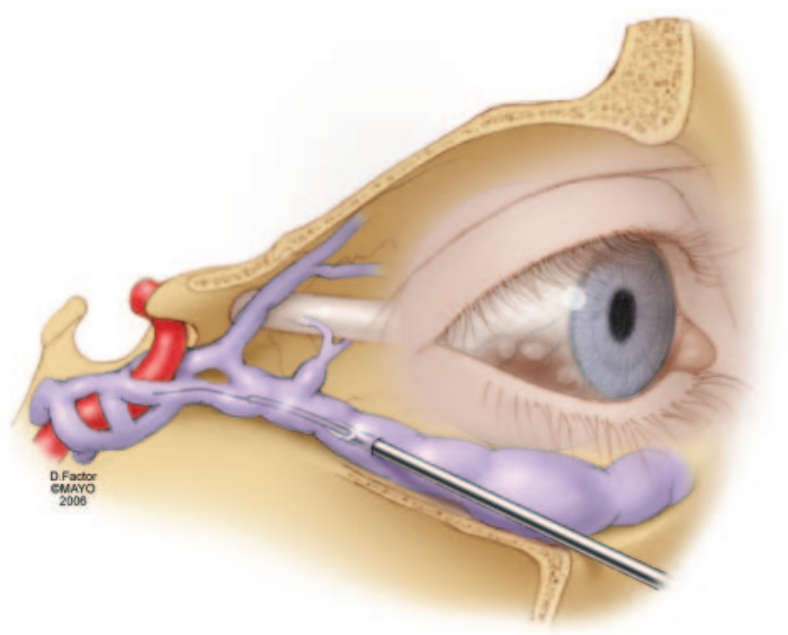

Fig 1. An illustration depicting the direct percutaneous approach to the cavernous sinus by way of the inferior ophthalmic vein. 

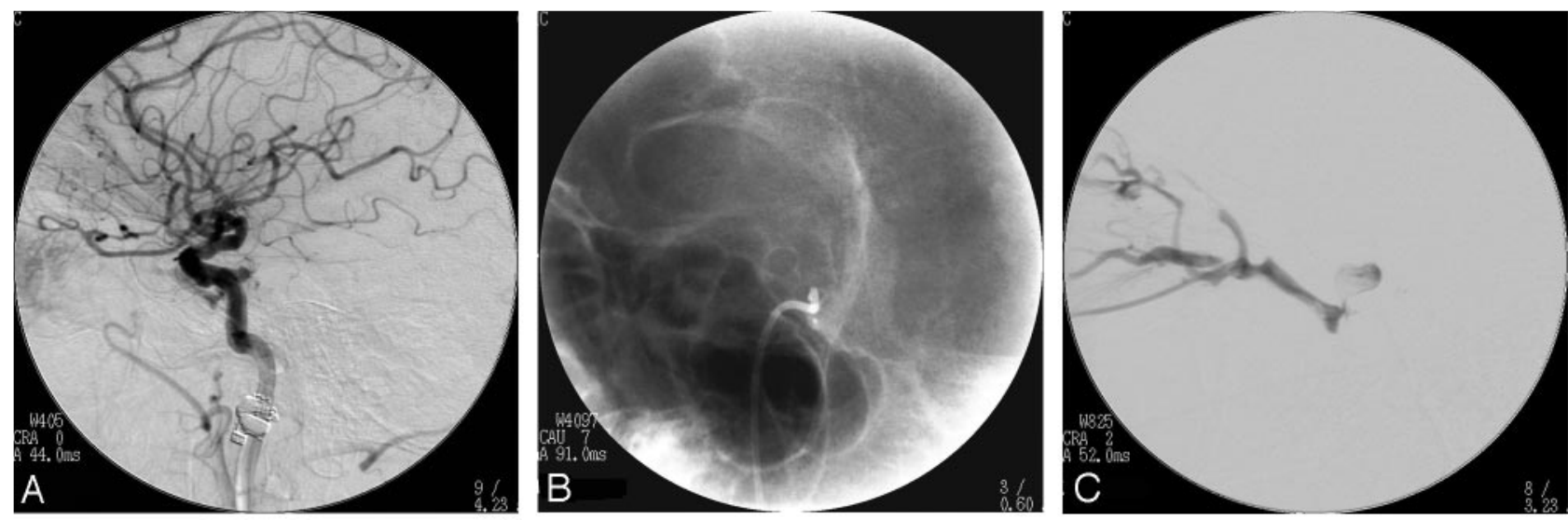

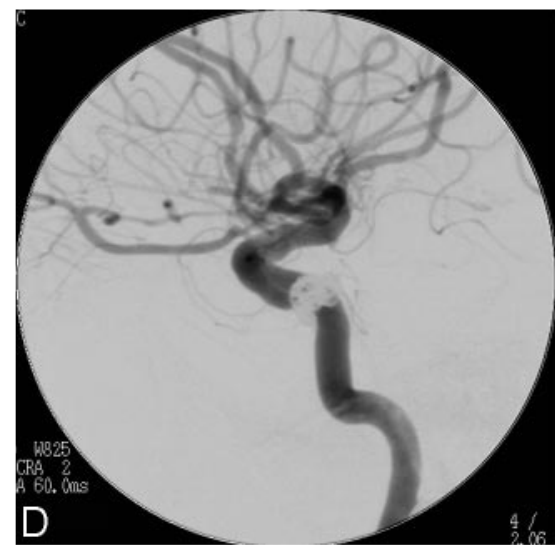

Fig 2. A, A left common carotid artery angiogram in the lateral projection reveals a CCF. $B, A$ transorbital oblique projection allows visualization of the SOF and optic canal. C, A cavernous sinus venogram after direct transorbital puncture via the inferior orbit is shown on a lateral view. D, A postprocedural angiogram shows successful embolization of the CCF.

and/or occlusion of the inferior petrosal sinuses. The remainder of the findings on the angiogram was unremarkable.

Following the successful embolization of the CCF (Fig 2), the patient's visual acuity had improved to 20/50 at 6 weeks after the procedure. There were no immediate or delayed complications from the embolization procedure. The proptosis and periorbital edema resolved, and she regained full mobility of her eye.

\section{Discussion}

Transvenous embolization has become the treatment of

\section{Results}

A total of 8 patients underwent successful embolization of their CCFs by using a direct transorbital cavernous sinus or IOV approach. In each patient, an obliteration of the CCF was obtained with a single procedure, documented by a postprocedural angiogram. In 1 patient, a transient decrease in visual acuity occurred in the eye ipsilateral to the puncture. The patient's vision improved markedly within 48 hours and was completely normal at a follow-up visit 9 months later. There were no other periprocedural complications. A representative patient with accompanying images is described in the following paragraph.

This patient was an 81-year-old woman, previously in excellent health, who awoke with nausea, dizziness, and left-eye proptosis with periorbital edema. Initially, there was not a change in her vision. During the ensuing days, her visual acuity deteriorated to 20/400, with a loss of central vision and the inability to discriminate color. She was also noted to have an afferent pupillary defect. Examination of her right eye yielded normal parameters, and she denied other neurologic symptoms.

A cerebral angiogram was obtained by using the methods described previously. The patient was noted to have an indirect CCF in the region of the left cavernous sinus that was fed exclusively from branches of the left ICA in the region of the meningohypophyseal trunk (Fig 2). Venous drainage was through a dorsal pouch along the posterolateral aspect of the horizontal segment of the left cavernous carotid artery. Slow venous egress was noted anteriorly through the inferior ophthalmic vein. No other venous outlet was observed. A transfemoral approach was unsuccessful due to poor visualization choice for cavernous sinus DAVFs. ${ }^{6,7}$ Alternative or additional treatment strategies are transarterial embolization, a combined surgical and endovascular approach, and the surgical exposure of the superior ophthalmic vein or cavernous sinus to perform direct embolization. Gamma knife alone or as an adjunct to particulate embolization is also a proved option for therapy. ${ }^{8}$ Cavernous sinus DAVFs are rarely life-threatening and usually have an indolent course, a low morbidity, and a high rate of spontaneous regression ${ }^{3}$; consequently, an initial conservative strategy may be appropriate. In those patients in whom a rapid deterioration of ocular symptoms and/or cortical venous drainage occurs, urgent interventional therapy is warranted.

The transfemoral venous approach has been and remains the most common and probably the safest means of access. ${ }^{4}$ When the transfemoral venous approach is not feasible, the transorbital direct puncture technique is a viable alternative. The venous drainage from the orbit is carried by way of the superior and inferior ophthalmic veins that empty into the cavernous sinus. Most anatomic reports support the notion that the main and constant draining vessel of the orbit is the superior ophthalmic vein that converges with the IOV to form a common venous confluence before entering the cavernous sinus. ${ }^{9,10}$ The inferior ophthalmic vein is often accompanied by other thin veins that empty into the venous confluence in most cases. In fact, the IOV may not be singular but rather a plexus of vessels that drain the inferior aspects of the orbit. ${ }^{9}$

We report a series of 8 patients in whom we were able to embolize their cavernous sinus DAVFs by using a percutaneous transorbital access pathway. This approach allows a safe and efficacious alternative to the transfemoral approach, particularly in the setting of pronounced anterior drainage in- 
volving the ophthalmic veins. In each case, our patients experienced alleviation of their symptoms without procedural complications. This technique allows an anatomically direct avenue to the cavernous sinus and, in our limited experience with this approach, does so without appreciable added risk to the patient. Furthermore, direct percutaneous transorbital cannulation of the cavernous sinus can be safely performed without a surgical exposure.

The potential risks of this procedure include orbital hemorrhage, nerve damage, laceration of the internal carotid artery resulting in direct CCF, globe puncture, and infection. Because of these potential risks, the transfemoral-transvenous approach is still the first choice for therapy in treating cavernous sinus DAVFs.

\section{Conclusions}

Cavernous sinus DAVFs can be safely and completely treated with endovascular techniques by using the direct transorbital puncture of the cavernous sinus or inferior ophthalmic vein. This approach can be maintained without undue complication to the patient and serves as a viable alternative when the jugular or inferior petrosal sinuses are not accessible. Other transvenous routes are also options but may necessitate surgical access for vein exposure.

\section{References}

1. Hamby WB. Carotid-cavernous fistula: report of 32 surgically treated cases and suggestions for definitive operation. J Neurosurg 1964;21:859-66

2. Hamby WB, Dohn DF. Carotid-cavernous fistulas: report of thirty-six cases and discussion of their management. Clin Neurosurg 1964;11:150-70

3. Ringer AJ, Salud L, Tomsick TA. Carotid cavernous fistulas: anatomy, classification, and treatment. Neurosurg Clin N Am 2005;16:279-95, viii

4. Cheng KM, Chan CM, Cheung YL. Transvenous embolisation of dural carotid-cavernous fistulas by multiple venous routes: a series of 27 cases. Acta Neuroch (Wein) 2003;145:17-29

5. Oono S, Matsui Y, Saito I, et al. Dural carotid-cavernous fistula successfully treated by embolization via inferior ophthalmic vein: case report. Neuro-Ophthalmology 1998;20:69

6. Halbach VV, Higashida RT, Dowd CF, et al. Cavernous internal carotid artery aneurysms treated with electrolytically detachable coils. J Neuroophthalmol 1997;17:231-39

7. Halbach VV, Higashida RT, Hieshima GB, et al. Dural fistulas involving the cavernous sinus: results of treatment in $\mathbf{3 0}$ patients. Radiology 1987;163: $437-42$

8. Pollock BE, Nichols DA, Garrity JA, et al. Stereotactic radiosurgery and particulate embolization for cavernous sinus dural arteriovenous fistulae. Neurosurgery 1999;45:459-66

9. Rhoton AL Jr. The cavernous sinus, the cavernous venous plexus, and the carotid collar. Neurosurgery 2002;51:S375-410

10. Yasuda A, Campero A, Martins C, et al. Microsurgical anatomy and approaches to the cavernous sinus. Neurosurgery 2005;56:4-27 